Draft VERSION NOVEMBER 12, 2018

Preprint typeset using ${ }^{A} \mathrm{~T}_{\mathrm{E}} \mathrm{X}$ style AASTeX6 v. 1.0

\title{
A 1420 MHZ CATALOG OF COMPACT SOURCES IN THE NORTHERN GALACTIC PLANE
}

\author{
A. R. TAYLOR \\ Inter-University Institute for Data Intensive Astronomy, and \\ Department of Astronomy, University of Cape Town \\ Department of Physics, University of the Western Cape \\ D. A. Leahy, T. Wenwu, C. Sunstrum \\ Department of Physics and Astronomy, University of Calgary \\ R. Kothes, T. L. Landecker, R. R Ransom, L. A. Higgs \\ Dominion Radio Astrophysical Observatory, Herzberg Programs in Astronomy and Astrophysics \\ National Research Council of Canada
}

\begin{abstract}
We present a catalog of compact sources of radio emission at $1420 \mathrm{MHz}$ in the northern Galactic plane from the Canadian Galactic Plane Survey. The catalog contains 72,787 compact sources with angular size less than $3^{\prime}$ within the Galactic longitude range $52^{\circ}<\ell<192^{\circ}$ down to a $5 \sigma$ detection level of $\sim 1.2$ mJy. Linear polarization properties are included for 12,368 sources with signals greater than $4 \sigma_{Q U}$ in the CGPS Stokes $Q$ and $U$ images at the position of the total intensity peak. We compare CGPS flux densities with cataloged flux densities in the Northern VLA Sky Survey catalog for 10,897 isolated unresolved sources with CGPS flux density greater than $4 \mathrm{mJy}$ to search for sources that show variable flux density on time scales of several years. We identify 146 candidate variables that exhibit high fractional variations between the two surveys. In addition we identify 13 candidate transient sources that have CGPS flux density above 10 mJy but are not detected in the NVSS.
\end{abstract}

Keywords: catalogs - radio continuum: general - surveys

\section{INTRODUCTION}

The Canadian Galactic Plane Survey (CGPS) mapped the principal constituents of the interstellar medium (ISM) with high angular resolution, inspired by the view that understanding the ISM requires knowledge of all its constituents. The Synthesis Telescope at the Dominion Radio Astrophysical Observatory (the DRAO ST) provided arcminute-resolution images of the radio continuum and atomic-hydrogen line emission. The CGPS is presented in Taylor et al. (2003), where details are also given of companion surveys, which imaged the molecular and dust constituents of the ISM. The DRAO ST is described in Landecker et al. (2000). Polarization imaging techniques and images of the diffuse Galactic polarized emission at $1420 \mathrm{MHz}$ are presented in Landecker et al.
(2010).

The CGPS DRAO radio continuum observations provide images of Stokes $I, Q$, and $U$ in four $7.5-\mathrm{MHz}$ subbands spanning $35 \mathrm{MHz}$, centred on $1420 \mathrm{MHz}$. The observations were carried out in three phases beginning in 1995 and ending in 2009. The sky coverage of each phase and observing dates are listed in Table 1. The Galactic plane was covered with a width in Galactic latitude of $9^{\circ}$, centred at $b=1^{\circ}$ to accommodate the warp of the Galactic disk. The longitude coverage was constrained by the southern declination limit of $\sim 20^{\circ}$, the range that could be effectively imaged by a linear East-West synthesis telescope array. The Phase II observations included an extension to higher latitudes $\left(b=17.5^{\circ}\right)$ over a restricted range of longitude. 
Table 1. Galactic Coordinate Coverage of the CGPS Observations

\begin{tabular}{lccc}
\hline \hline Phase & $\begin{array}{c}\text { Galactic Latitude } \\
\text { (degrees) }\end{array}$ & $\begin{array}{c}\text { Galactic Longitude } \\
\text { (degrees) }\end{array}$ & Dates \\
\hline Phase I & $74<\ell<147$ & $-3.6<b<5.6$ & Apr 1995- Jun 2000 \\
Phase II & $64<\ell<74$ & $-3.6<b<5.6$ & Feb 2000- Apr 2004 \\
& $147<\ell<175$ & $-3.6<b<5.6$ & \\
& $100<\ell<117$ & $5.6<b<17.5$ & \\
Phase III & $52<\ell<64$ & $-3.6<b<5.6$ & Nov 2003- Feb 2009 \\
& $175<\ell<192$ & $-3.6<b<5.6$ & \\
& & & \\
\hline
\end{tabular}

At radio wavelengths the plane of the Galaxy is characterized by strong and highly-structured extended emission from the ionized and relativistic plasma of the ISM. The presence of such complex emission structure on all angular scales presents challenges for interferometric observations. To minimize imaging artefacts the CGPS observations fully sample the two-dimensional spatial frequency distribution of the sky from zero spacing up to the maximum baseline of $617 \mathrm{~m}$, and directiondependent self-calibration is used to remove the effects of sources outside of the primary beams of the antennas. The CGPS 1420-MHz images are thus noise-limited images largely free of imaging artefacts over most of the survey area. The band-integrated images have a $1 \sigma$ RMS noise as low as $0.18 \mathrm{mJy}$. These data therefore provide an opportunity to create a sensitive catalog of radio continuum sources over a large area of the northern Galactic plane, including both total intensity and linear polarization.

In this paper we present the CGPS $1420-\mathrm{MHz}$ compact source catalog covering 1464 square degrees and spanning a range of $140^{\circ}$ of Galactic longitude between $52^{\circ}<\ell<192^{\circ}$. The catalog is presented and described in Section 2, including flux densities in Stokes $I$ and polarized intensities. We compare the CGPS compact source catalog with the Northern VLA Sky Survey (NVSS) catalog (Condon et al. 1998) in Section 3. Since the two surveys have comparable resolution and sensitivity, but were separated in time, we are able to carry out a simple analysis for variability. We identify a number of variable sources and transient candidates in (Section 3.3).

\section{THE COMPACT SOURCES CATALOG}

\subsection{The Source List}

The CGPS DRAO image products are $5.12^{\circ} \times 5.12^{\circ}$ images mosaicked from overlapping Synthesis Telescope fields. The images are available through the Cana- dian Astronomy Data Centre ${ }^{1}$. The complete survey is comprised of 85 such mosaics. We constructed the radio source catalog from these images using an automated source finding algorithm, FINDSRC, developed as part of the DRAO export software package (Higgs et al. 1997). FINDSRC uses a wavelet filter convolution to identify compact source candidates in the image. This convolution effectively filters out emission structures larger than a few beam sizes. A second DRAO software program, FLUXFIT, reads in the candidates identified in the filtered image by FINDSRC and fits the signals in the original, unfiltered image with a twodimensional Gaussian function plus a "twisted" plane fit to the local background level. To avoid the potential problem of nearby sources within the fitting region, if a nearby sources is detected, FINDSRC expands the area of the region to include that source and carries out a simultaneous multiple Gaussian fit to all sources in the region. The output of FINDSRC are fit parameters and errors for the peak and integrated flux density of the source above the local background, as well as the major and minor axes and the major axis position angle of the best-fit Gaussian approximation to the source response function. For each source an RMS background level was calculated from the variance in the image around the twisted plane background fit. We retained a source in the catalog if the peak amplitude of the best-fit Gaussian is greater than five times the background RMS.

Following the automated source extraction, an annotation file marking the position of each detected source was overlaid on the mosaic images and each image was examined visually to identify spurious detections arising, for example, from knots on filaments of highly structured diffuse emission in the Galactic Plane. On a few occasions a source was missed by the automated

1 www.cadc-ccda.hia-iha.nrc-cnrc.gc.ca/en/cgps 
detection algorithm. In this case Gaussian fits were run "manually". The final source list contains 72,787 sources down to a minimum Stokes $I$ peak intensity of

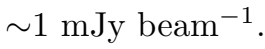

Because the DRAO ST is an East-West aperture synthesis array, the major axis of the synthesized beam has position angle of zero (oriented North-South) and its dimension is a function of declination. The angular resolution of the ST observations is given by

$$
\mathrm{b}_{\text {major }} \times \mathrm{b}_{\text {minor }}=49^{\prime \prime} \operatorname{cosec} \delta \times 49^{\prime \prime}
$$

where $b_{\text {major }}$ and $b_{\text {minor }}$ are respectively in the Declination and Right Ascension directions. A Gaussian synthesized beam is deconvolved from the fitted twodimensional Gaussian to estimate the observed angular size of each source. A source is considered unresolved in either the major or minor axis dimension if the deconvolved angular size is less than 3 times the $1 \sigma$ error on the solid angle. Unresolved dimensions are listed in the catalog with zero dimension.

A measure of the polarized intensity for each source was obtained from intensity of the Stokes $Q$ and $U$ images at the Stokes $I$ position of each source after removing a mean background level determined in a $25 \times 25$ pixel region around each source. A provisional polarization detection was flagged if either the $Q$ or $U$ signal was greater than four times the local RMS, $\sigma_{Q}$ and $\sigma_{U}$, in the region surrounding the source. For provisional detections an estimate, $\hat{p}_{o}$, of the intrinsic polarized intensity, $p_{o}$, was calculated as

$$
\hat{p}_{o}=\sqrt{p^{2}-\sigma_{\mathrm{QU}}^{2}},
$$

which is good estimator of the polarized intensity when $p / \sigma>4$ (Simmons \& Stewart 1985). A source was retained as a polarized detection if in addition $\hat{p}_{o}>\epsilon_{p} S_{p}$, where $\epsilon_{p}$ is the instrumental polarization error, taken to be $0.3 \%$ (Taylor et al. 2003), and $S_{p}$ is the peak Stokes $I$ intensity. The error on the polarized intensity is given by

$$
\sigma_{\hat{p}_{o}}=\sqrt{2 \sigma_{\mathrm{QU}}^{2}+\left(\epsilon_{p} S_{p}\right)^{2}} .
$$

We detect polarized signals from 12,368 sources. The polarized intensity should be treated with caution for resolved sources. The polarized intensity structure for extended sources is not necessarily the same as that of the total intensity. In such cases the polarized intensity at the position of the total intensity peak may not represent the true peak of $Q$ and $U$. Those interested in the polarized emission from extended sources are advised to examine the CGPS images directly.

Table 2 lists the first thirty entries of the CGPS 1420 MHz catalog. The columns contain: (1) the source name CGPS JHHMMSS+DDMMSS, where HHMMSS are the hours and minutes and seconds of time for Right Ascension, and DDMMSS are the degrees, minutes and seconds of arc of Declination (both in J2000), (2) the J2000 Right Ascension (degrees) and error (seconds), (3) the J2000 Declination (degrees) and error (arcseconds), (4) the integrated Stokes $I$ flux density and error (mJy), (5) the peak Stokes $I$ intensity and error (mJy/beam), (6) the average rms around the position of the source in the $Q$ and $U$ images (mJy), (7) the bias corrected polarized intensity in the case of detected polarized emission (mJy/beam), (8) the polarization position angle (degrees), (9 and 10) the deconvolved dimensions of the major and minor axes of the Stokes $I$ emission with errors (arc seconds), and (11) the position angle of the source major axis (degrees) with respect to north. The deconvolved source dimensions are listed as zero if the source is classed as unresolved. The beam dimensions at the location of the source can be calculated using Equation 1. All errors are $1 \sigma$.

The complete version of Table 2 is available in machine-readable format through the CGPS data repos-

\begin{tabular}{|c|c|c|c|c|c|c|c|c|c|c|}
\hline Name & $\mathrm{RA}(\mathrm{J} 2000)$ & $\mathrm{DEC}(\mathrm{J} 2000)$ & $\begin{array}{c}\mathrm{S}_{\mathrm{I}} \\
(\mathrm{mJy})\end{array}$ & $\begin{array}{c}\mathrm{S}_{\mathrm{P}} \\
(\mathrm{mJy} / \mathrm{bm})\end{array}$ & $\begin{array}{c}\sigma_{Q U} \\
(\mathrm{mJy})\end{array}$ & $\begin{array}{c}\mathrm{P} \\
(\mathrm{mJy} / \mathrm{bm})\end{array}$ & $\begin{array}{c}\text { Pol PA } \\
\text { (०) }\end{array}$ & \multicolumn{2}{|c|}{ (arc-seconds) } & $\begin{array}{l}\text { PA } \\
\text { (0) }\end{array}$ \\
\hline CGPS J000001+610821 & $0.00508 \pm 0.47$ & $61.13939 \pm 4.6$ & $2.6 \pm 0.31$ & $2.6 \pm 0.31$ & 0.27 & $0.0 \pm 0.00$ & 0 & $0.0 \pm 0.0$ & $0.0 \pm 0.0$ & 0 \\
\hline CGPS J000002+670758 & $0.00887 \pm 0.30$ & $67.13281 \pm 0.7$ & $37.1 \pm 1.24$ & $30.7 \pm 1.12$ & 0.31 & $2.2 \pm 0.32$ & 69 & $29.3 \pm 0.8$ & $15.3 \pm 1.4$ & 76 \\
\hline CGPS J000002+605312 & $0.00996 \pm 0.66$ & $60.88678 \pm 13.4$ & $1.2 \pm 0.27$ & $1.2 \pm 0.27$ & 0.34 & $0.0 \pm 0.00$ & 0 & $0.0 \pm 0.0$ & $0.0 \pm 0.0$ & 0 \\
\hline CGPS J000002+620952 & $0.01162 \pm 1.07$ & $62.16464 \pm 6.0$ & $1.2 \pm 0.25$ & $1.2 \pm 0.25$ & 0.30 & $0.0 \pm 0.00$ & 0 & $0.0 \pm 0.0$ & $0.0 \pm 0.0$ & 0 \\
\hline CGPS J000003+625231 & $0.01650 \pm 0.40$ & $62.87531 \pm 2.2$ & $8.1 \pm 0.47$ & $8.1 \pm 0.47$ & 0.30 & $1.6 \pm 0.29$ & -72 & $0.0 \pm 0.0$ & $0.0 \pm 0.0$ & 0 \\
\hline CGPS J000005+670622 & $0.02346 \pm 0.57$ & $67.10622 \pm 6.7$ & $2.0 \pm 0.28$ & $2.0 \pm 0.28$ & 0.32 & $0.0 \pm 0.00$ & 0 & $0.0 \pm 0.0$ & $0.0 \pm 0.0$ & 0 \\
\hline CGPS J000006+592611 & $0.02567 \pm 0.48$ & $59.43644 \pm 4.6$ & $2.6 \pm 0.32$ & $2.6 \pm 0.32$ & 0.36 & $0.0 \pm 0.00$ & 0 & $0.0 \pm 0.0$ & $0.0 \pm 0.0$ & 0 \\
\hline CGPS J000007+624215 & $0.03021 \pm 0.53$ & $62.70439 \pm 3.9$ & $3.2 \pm 0.31$ & $3.2 \pm 0.31$ & 0.34 & $0.0 \pm 0.00$ & 0 & $0.0 \pm 0.0$ & $0.0 \pm 0.0$ & 0 \\
\hline CGPS J000007+660841 & $0.03133 \pm 0.31$ & $66.14472 \pm 0.9$ & $10.7 \pm 0.49$ & $9.9 \pm 0.40$ & 0.21 & $1.0 \pm 0.22$ & 26 & $19.8 \pm 2.0$ & $0.0 \pm 0.0$ & 66 \\
\hline CGPS J000015+624330 & $0.06450 \pm 0.32$ & $62.72511 \pm 1.3$ & $11.7 \pm 0.47$ & $11.7 \pm 0.47$ & 0.30 & $0.0 \pm 0.00$ & 0 & $0.0 \pm 0.0$ & $0.0 \pm 0.0$ & 0 \\
\hline CGPS J000015+611227 & $0.06525 \pm 0.57$ & $61.20769 \pm 4.1$ & $2.9 \pm 0.28$ & $2.9 \pm 0.28$ & 0.30 & $0.0 \pm 0.00$ & 0 & $0.0 \pm 0.0$ & $0.0 \pm 0.0$ & 0 \\
\hline CGPS J000015+625015 & $0.06550 \pm 0.30$ & $62.83761 \pm 0.7$ & $78.0 \pm 2.59$ & $48.9 \pm 2.31$ & 0.37 & $0.0 \pm 0.00$ & 0 & $57.0 \pm 0.8$ & $19.5 \pm 1.3$ & 128 \\
\hline CGPS J000016+621817 & $0.06904 \pm 0.66$ & $62.30492 \pm 7.2$ & $3.1 \pm 0.49$ & $3.1 \pm 0.49$ & 0.35 & $0.0 \pm 0.00$ & 0 & $0.0 \pm 0.0$ & $0.0 \pm 0.0$ & 0 \\
\hline
\end{tabular}
itory at the Canadian Astronomy Data Centre.

Table 2. CGPS 1420 MHz Source Catalog 
Table 2 (continued)

\begin{tabular}{|c|c|c|c|c|c|c|c|c|c|c|}
\hline Name & $\mathrm{RA}(\mathrm{J} 2000)$ & $\mathrm{DEC}(\mathrm{J} 2000)$ & $\begin{array}{c}\mathrm{S}_{\mathrm{I}} \\
(\mathrm{mJy})\end{array}$ & $\begin{array}{c}\mathrm{S}_{\mathrm{P}} \\
(\mathrm{mJy} / \mathrm{bm})\end{array}$ & $\begin{array}{c}\sigma_{Q U} \\
(\mathrm{mJy})\end{array}$ & $\begin{array}{c}\mathrm{P} \\
(\mathrm{mJy} / \mathrm{bm})\end{array}$ & $\begin{array}{c}\text { Pol PA } \\
\text { (o) }\end{array}$ & $\begin{array}{l}\theta_{\mathrm{maj}} \\
\quad(\operatorname{arc}-\mathrm{s}\end{array}$ & $\begin{array}{l}\theta_{\min } \\
\text { conds) }\end{array}$ & $\begin{array}{l}\text { PA } \\
\text { (0) }\end{array}$ \\
\hline CGPS J000017+631526 & $0.07125 \pm 0.53$ & $63.25731 \pm 4.3$ & $3.2 \pm 0.52$ & $2.3 \pm 0.28$ & 0.25 & $0.0 \pm 0.00$ & 0 & $39.0 \pm 8.4$ & $23.7 \pm 0.2$ & 144 \\
\hline CGPS J000017+605635 & $0.07271 \pm 0.30$ & $60.94317 \pm 0.7$ & $29.8 \pm 1.03$ & $26.7 \pm 0.91$ & 0.30 & $0.0 \pm 0.00$ & 0 & $21.3 \pm 1.1$ & $12.2 \pm 2.1$ & 97 \\
\hline CGPS J000018+622221 & $0.07662 \pm 0.32$ & $62.37258 \pm 1.3$ & $11.2 \pm 0.47$ & $11.2 \pm 0.47$ & 0.29 & $0.0 \pm 0.00$ & 0 & $0.0 \pm 0.0$ & $0.0 \pm 0.0$ & 0 \\
\hline CGPS J000018+605251 & $0.07683 \pm 0.35$ & $60.88100 \pm 2.5$ & $4.5 \pm 0.31$ & $4.5 \pm 0.31$ & 0.32 & $0.0 \pm 0.00$ & 0 & $0.0 \pm 0.0$ & $0.0 \pm 0.0$ & 0 \\
\hline CGPS J000019+630951 & $0.08088 \pm 0.30$ & $63.16419 \pm 0.7$ & $21.2 \pm 0.78$ & $19.7 \pm 0.67$ & 0.27 & $0.0 \pm 0.00$ & 0 & $19.3 \pm 1.6$ & $8.5 \pm 2.8$ & 168 \\
\hline CGPS J000020+611404 & $0.08608 \pm 0.59$ & $61.23453 \pm 2.9$ & $3.1 \pm 0.28$ & $3.1 \pm 0.28$ & 0.23 & $0.0 \pm 0.00$ & 0 & $0.0 \pm 0.0$ & $0.0 \pm 0.0$ & 0 \\
\hline CGPS J000021+610428 & $0.08862 \pm 0.49$ & $61.07444 \pm 6.3$ & $1.9 \pm 0.27$ & $1.9 \pm 0.27$ & 0.30 & $0.0 \pm 0.00$ & 0 & $0.0 \pm 0.0$ & $0.0 \pm 0.0$ & 0 \\
\hline CGPS J000021+661831 & $0.08908 \pm 0.54$ & $66.30878 \pm 8.2$ & $2.2 \pm 0.51$ & $1.2 \pm 0.23$ & 0.20 & $0.0 \pm 0.00$ & 0 & $79.1 \pm 0.7$ & $0.0 \pm 0.0$ & 70 \\
\hline CGPS J000023+642554 & $0.09617 \pm 0.31$ & $64.43186 \pm 0.9$ & $19.6 \pm 0.82$ & $16.1 \pm 0.64$ & 0.31 & $0.0 \pm 0.00$ & 0 & $28.0 \pm 1.7$ & $20.0 \pm 1.9$ & 23 \\
\hline CGPS J000024+621506 & $0.10054 \pm 0.30$ & $62.25178 \pm 0.6$ & $336.8 \pm 9.91$ & $277.4 \pm 9.84$ & 0.35 & $10.7 \pm 0.90$ & 65 & $27.2 \pm 0.3$ & $20.6 \pm 0.4$ & 64 \\
\hline CGPS J000025+641845 & $0.10438 \pm 0.43$ & $64.31256 \pm 2.9$ & $4.7 \pm 0.31$ & $4.7 \pm 0.31$ & 0.33 & $0.0 \pm 0.00$ & 0 & $0.0 \pm 0.0$ & $0.0 \pm 0.0$ & 0 \\
\hline CGPS J000026+641327 & $0.11067 \pm 0.65$ & $64.22419 \pm 6.3$ & $1.6 \pm 0.29$ & $1.6 \pm 0.29$ & 0.30 & $0.0 \pm 0.00$ & 0 & $0.0 \pm 0.0$ & $0.0 \pm 0.0$ & 0 \\
\hline CGPS J000027+632556 & $0.11287 \pm 0.43$ & $63.43236 \pm 3.4$ & $2.8 \pm 0.27$ & $2.8 \pm 0.27$ & 0.33 & $0.0 \pm 0.00$ & 0 & $0.0 \pm 0.0$ & $0.0 \pm 0.0$ & 0 \\
\hline CGPS J000027+595049 & $0.11488 \pm 0.32$ & $59.84711 \pm 1.3$ & $6.9 \pm 0.32$ & $6.9 \pm 0.32$ & 0.43 & $0.0 \pm 0.00$ & 0 & $0.0 \pm 0.0$ & $0.0 \pm 0.0$ & 0 \\
\hline CGPS J000028+661149 & $0.11937 \pm 0.32$ & $66.19694 \pm 1.1$ & $7.8 \pm 0.45$ & $6.6 \pm 0.32$ & 0.23 & $0.0 \pm 0.00$ & 0 & $23.3 \pm 2.3$ & $19.4 \pm 2.9$ & 66 \\
\hline CGPS J000029+595722 & $0.12246 \pm 0.33$ & $59.95636 \pm 1.8$ & $5.8 \pm 0.32$ & $5.8 \pm 0.32$ & 0.42 & $0.0 \pm 0.00$ & 0 & $0.0 \pm 0.0$ & $0.0 \pm 0.0$ & 0 \\
\hline CGPS J000029+593426 & $0.12429 \pm 0.30$ & $59.57394 \pm 0.7$ & $28.8 \pm 1.01$ & $24.1 \pm 0.88$ & 0.29 & $0.0 \pm 0.00$ & 0 & $25.9 \pm 1.0$ & $20.8 \pm 1.3$ & 125 \\
\hline
\end{tabular}

\section{COMPARISON TO THE NVSS}

\subsection{Position and Flux Densities}

Our survey is comparable in resolution and sensitivity to the NRAO VLA Sky Survey (Condon et al. 1998); the flux density scale and source positions of the CGPS at $1420 \mathrm{MHz}$ were tied to the NVSS. Figure 1 shows the distribution of source flux densities for the CGPS and for the NVSS over the region of the CGPS survey. These curves give an indication of the relative depths of the catalogs. The NVSS flux distribution peaks at $3 \mathrm{mJy}$ and drops to zero by $2 \mathrm{mJy}$. The CGPS source numbers continue to increase to $2 \mathrm{mJy}$, with $20 \%$ of the sources having flux density below $2 \mathrm{mJy}$. This is consistent with the relative noise levels of $\sim 0.45 \mathrm{mJy}$ for the NVSS and $\sim 0.24 \mathrm{mJy}$ for the CGPS. Moreover, the CGPS, with an almost filled $u-v$ plane coverage, has a much more uniform image background noise distribution, with very little contamination from sidelobes of the synthesized beam. Given the strong and highly structured diffuse emission that is ubiquitous in the plane of the Galaxy, the depth of the survey will vary significantly with location in the plane, but should be complete to about 2 $\mathrm{mJy}$ in quiet regions.

From the 72,787 sources in the CGPS catalog we identified 52,876 that have counterparts in the NVSS within $60^{\prime \prime}$ of the CGPS position. Figure 2 shows a plot of the position differences between the CGPS and NVSS catalog sources for 34,449 sources with CGPS flux density above $5 \mathrm{mJy}$. The individual CGPS images are registered in position and flux density against the NVSS images using a small number of strong sources within each field (Taylor et al. 2003). Using the very large number of sources in the catalog comparison we measure an inverse signal-to-noise weighted mean difference between the two catalogs of $\Delta \alpha=0.011 \pm 0.003^{\prime \prime}$ and $\Delta \delta=-0.024 \pm 0.005^{\prime \prime}$. The difference is small, but significant relative to the error. To make the CGPS catalog positions formally consistent on average with the NVSS to within $0.005^{\prime \prime}$, we have corrected for this small difference for the source coordinates listed in Table 2.

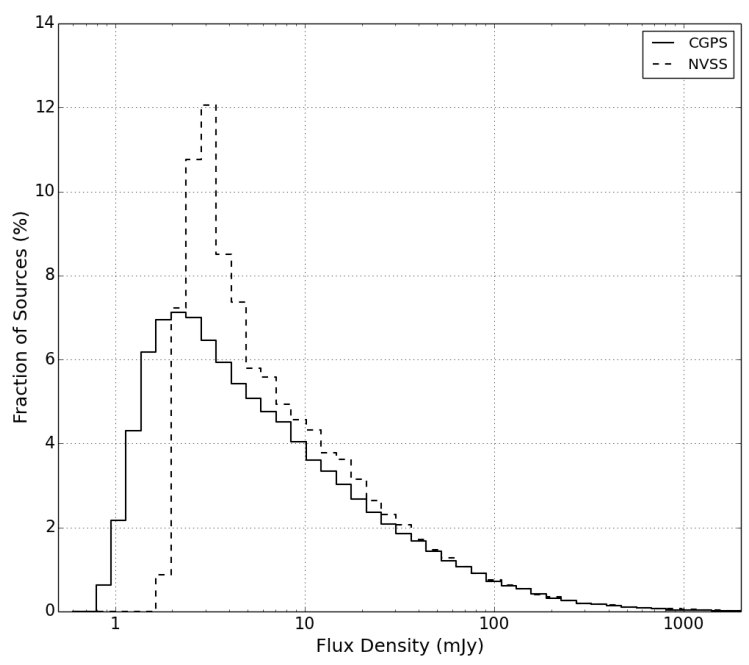

Figure 1. The distribution of integrated flux densities for the CGPS sources (solid histogram) and for the NVSS catalog over the CGPS survey area (dashed line). 


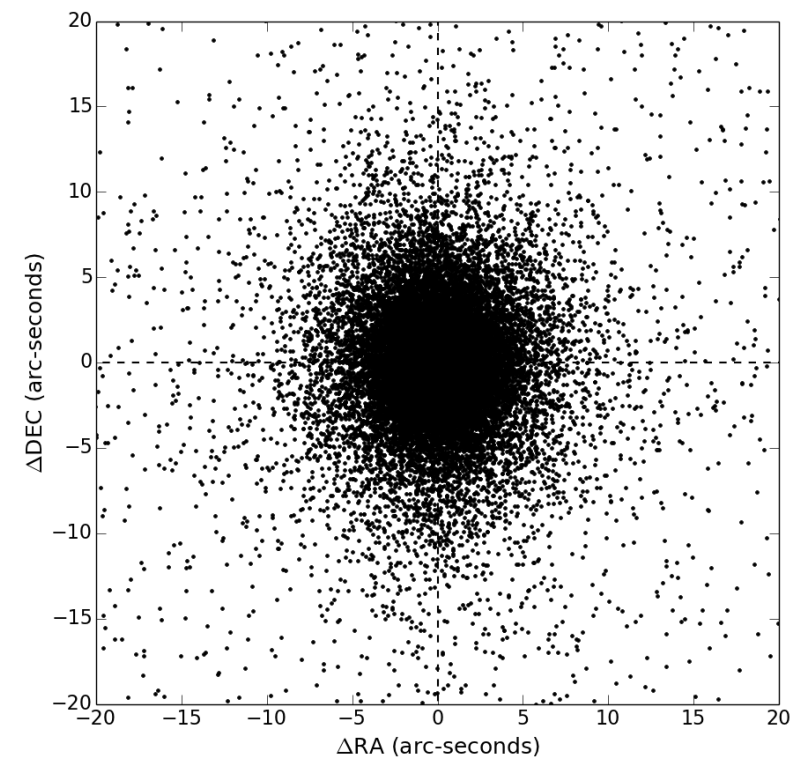

Figure 2. The differences in right ascension and declination between the CGPS and NVSS catalog positions.

To compare the CGPS and NVSS flux densities we calculate for all sources detected in both surveys the fractional difference in flux density, $F$, defined as

$$
F=2\left(\frac{S_{\mathrm{CGPS}}-S_{\mathrm{NVSS}}}{S_{\mathrm{CGPS}}+S_{\mathrm{NVSS}}}\right) .
$$

The result is plotted in Figure 3 for sources detected with a position difference between the two surveys of less than 10", and CGPS major-axis diameter less than $20^{\prime \prime}$. The solid line in the figure, that delimits the upper bound of the data points, shows the selection effect of a 2 mJy detection threshold in the NVSS, which is approximately the NVSS minimum detectable flux density. The absence of points above this line is due to noise variations in the NVSS data reducing the NVSS signals of CGPS sources below the NVSS detection limit. We used 370 unresolved sources with $S_{\mathrm{CGPS}}>40 \mathrm{mJy}$ and position differences less than $10^{\prime \prime}$ to derive a median fractional difference of $\langle F\rangle=-0.0289 \pm 0.005$. The raw CGPS flux densities are in the median $2.9 \%$ lower than the NVSS catalog flux densities. We have corrected for this in the CGPS catalog. The CGPS and NVSS flux density scales are thus aligned with an error of $0.5 \%$.

\subsection{Polarization}

Figure 4 shows the fractional difference in peak polarized flux density, $F_{p}$, between the CGPS and NVSS for polarized sources in the CGPS. The data show that for the CGPS polarized intensities are on average substantially larger than the NVSS. This arises from the

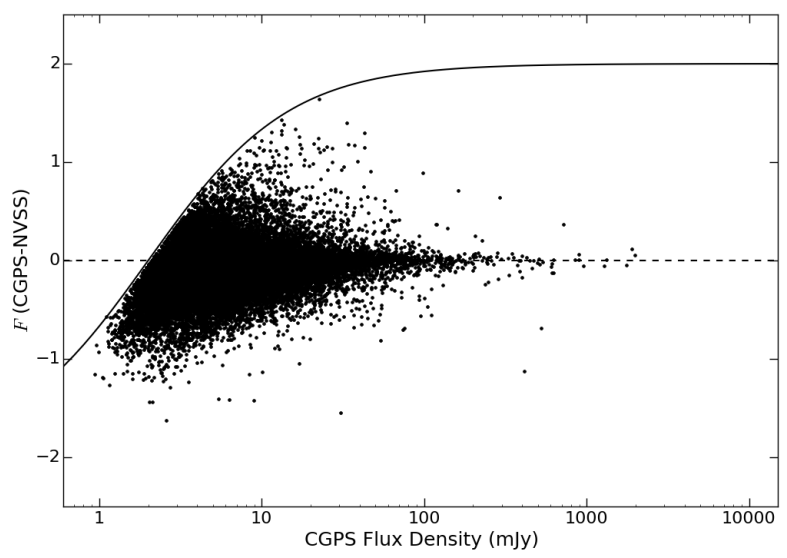

Figure 3. The fractional difference in flux density between the CGPS and NVSS for all CGPS sources with a counterpart in the NVSS catalog within $10^{\prime \prime}$. The solid line shows the effect of a detection threshold in the NVSS flux densities of $2 \mathrm{mJy}$.

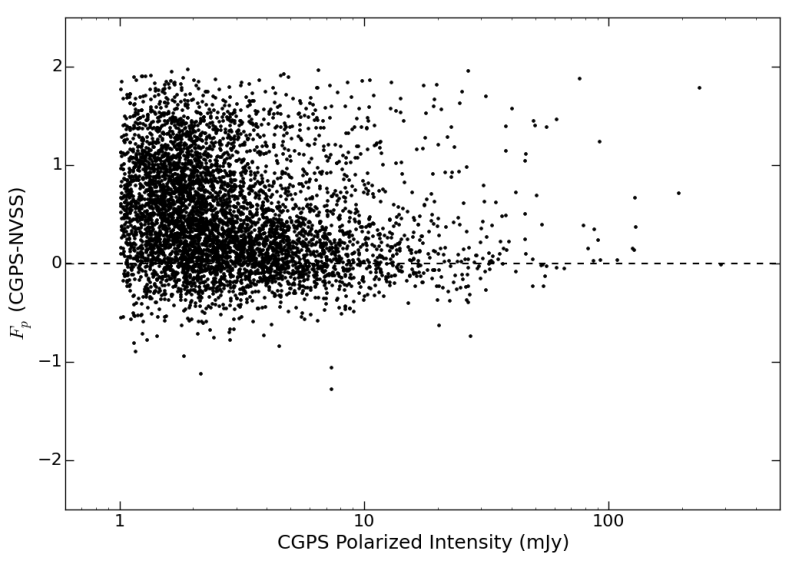

Figure 4. The fractional difference in peak polarized intensity between CGPS and NVSS for all sources detected in polarization in the CGPS having NVSS counterparts within $10^{\prime \prime}$.

effect on the NVSS polarized intensities of the high levels of Faraday Rotation from propagation through the magneto-ionic medium of the Galaxy at the low Galactic latitudes of the CGPS.

As noted by Condon et al. (1998), the widely separated dual-band structure of the NVSS survey produced significant depolarization in the band-average polarized intensities for Rotation Measure (RM) magnitudes larger than about $100 \mathrm{rad} \mathrm{m}^{-1}$. Over the area of the CGPS, RM values of this order and larger are common (Brown et al. 2003). The depolarization effect is illustrated by the data points in Figure 5, which show, as a function of source RM, the mean ratio of NVSS 


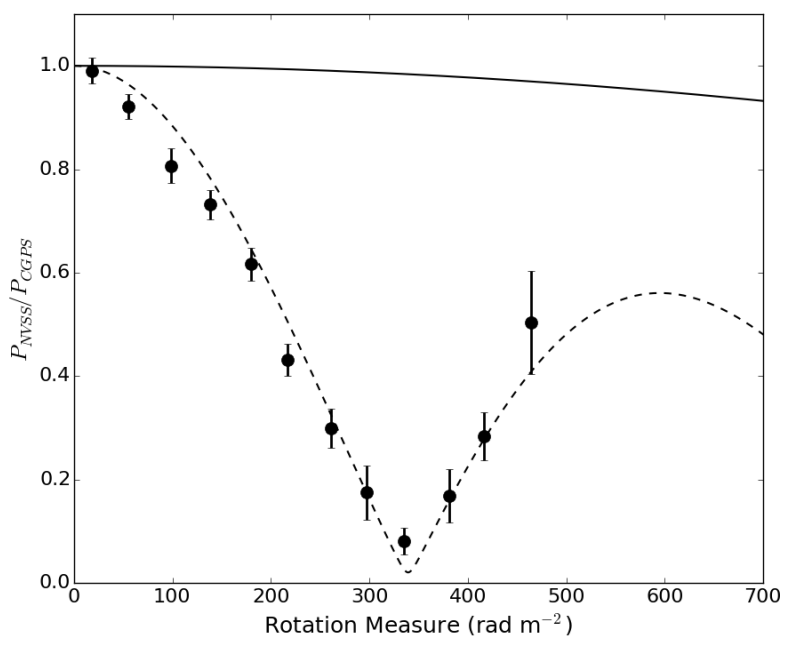

Figure 5. Comparison of NVSS and CGPS polarized intensity as a function of Rotation Measure. The dashed line shows the expected fractional bandwidth depolarization for the NVSS band structure. The solid line shows the same quantity for the CGPS. The data points are average values of the ratio of NVSS to CGPS polarized intensity as a function of source Rotation Measure. The NVSS bandwidth depolarization accounts for the systematically higher CGPS polarizations shown in Figure 4.

to CGPS peak polarized intensity for CGPS polarized sources having $p_{\mathrm{CGPS}}>10 \mathrm{mJy}$. Rotation Measures were derived from the two-bands of the NVSS data (Taylor et al. 2009). The ratio recovers the theoretical depolarization curve of the NVSS band structure (shown by the dashed line in the Figure). The narrower band CGPS data suffer less than $10 \%$ depolarization up to Rotation Meaures of $\sim 1000 \mathrm{rad} \mathrm{m}^{-2}$. The CGPS polarization data thus provide a low-latitude complement to the NVSS polarization catalog.

\subsection{Variable and Transient Sources}

The CGPS observations were taken over the period 1995 to 2009, while the NVSS data were taken between 1993 and 1996. The similar resolutions and sensitivities of the two surveys allow for the identification of sources in the plane of the Galaxy that have variable flux density over time intervals of a few to several years to sensitivities of a few mJy by comparison of the catalog data.

Several candidate variable sources are visible in Figure 3 as outliers to the noise-broadened distribution of $F$. However, some of these are spurious, resulting from differences between the NVSS and CGPS algorithms for identifying and fitting sources as either close double or extended. In some cases objects fit as single extended sources in the NVSS are fit as multiples in the CGPS, and vice-versa. Extended single component sources that are split into multiple components in the other catalog, will have very different flux densities, and each component will be offset in position from the single fit. To remove this effect from the variability analysis, we have restricted the search for variability to unresolved CGPS sources that are isolated from their nearest CGPS neighbour by at least $90^{\prime \prime}$, and whose NVSS counterpart also classed as unresolved and agrees in position within $5^{\prime \prime}$. We also restrict the analysis to sources with $S_{\text {CGPS }}>4.0$ mJy. There are 10,897 CGPS sources that meet these criteria.

As shown in Equation 4, the quantity $F$ measures the change in flux density between CGPS and NVSS as a fraction of the mean flux density measured between the two surveys. A change in flux density by a factor of 2 results in $F= \pm 0.67$. The significance of the change is quantified by a comparison to the uncertainty on $F$. We calculate this uncertainty, $\sigma_{F}$, by propagating the errors on the individual flux densities $S_{\mathrm{CGPS}}$ and $S_{\mathrm{NVSS}}$. We then identify as variables sources those for which

$$
V=\frac{F-F_{\mathrm{MED}}}{\sigma_{F}}>4
$$

where $F_{\mathrm{MED}}$ is the median value of $F$ for population. In the absence of variable sources, if $\sigma_{F}$ is an accurate measure of the error, the variance in $F$ will be determined by noise alone and the distribution of $V$ over the ensemble of sources will be Gaussian with unit standard deviation. Figure 6 shows the distribution of $V$ for the CGPS variable search sample. The central peak of the distribution is well fit by the Gaussian. Evidence for a population of variable sources appears as excess over the Gaussian distribution in the wings, beginning at about $|V| \sim 2$. However, we take a conservative approach and class as a variable sources those for which $|V|>4$. Of the 10,897 sources examined, $146(1.3 \%)$ satisfy this criterion. For a Gaussian distribution we expect less than 1 object at $|V|>4$ by chance.

Figure 6 shows an asymmetry in the tail of the distribution, with more objects with high positive $V$. There are 111 sources with $V>4$, compared to 35 with $V<-4$. This is a result of the threshold in $S_{\mathrm{CGPS}}$, which results in a bias against large negative values of $F$. A source with $S_{\mathrm{CGPS}}>4$ mJy and $F<-1.0$ must have $S_{\text {NVSS }}>12 \mathrm{mJy}$, which represents a small fraction of the NVSS sources. Conversely, $F>+1.0$ requires $S_{\text {NVSS }}>1.25 \mathrm{mJy}$, sampling the entire NVSS catalog.

While not a complete statistical study for sources of variable emission in the Galactic plane at $1420 \mathrm{MHz}$, this simple comparison does identify a small sample of isolated, unresolved radio sources whose flux density exhibits a large fractional change between the two catalogs. The resulting list of variable source candidates is given in Table 3. 


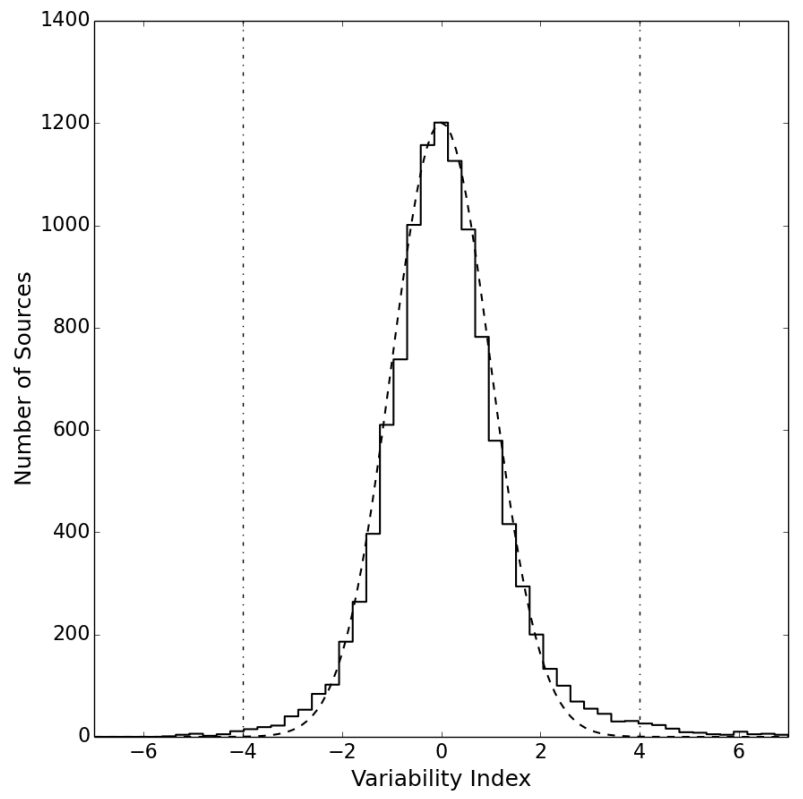

Figure 6. Distribution of variability indices, $V$. The solid histogram is for sources with CGPS flux density greater than $4 \mathrm{mJy}$. The dashed curve is a Gaussian distribution with standard deviation of unity normalised to the same peak as the histogram. The vertical dot-dashed lines indicate the limit of $V$ for classification as a variable.

Table 3. Variable Sources Detected in CGPS and NVSS

\begin{tabular}{|c|c|c|c|c|}
\hline Name & $\begin{array}{c}\mathrm{S}_{\mathrm{CGPS}} \\
(\mathrm{mJy})\end{array}$ & $\begin{array}{l}\mathrm{S}_{\mathrm{NVSS}} \\
(\mathrm{mJy})\end{array}$ & $\mathrm{F}$ & $\mathrm{V}$ \\
\hline CGPS J000636+673019 & $14.8 \pm 0.6$ & $6.9 \pm 0.5$ & 0.73 & 5.38 \\
\hline CGPS J000728+671954 & $38.2 \pm 1.7$ & $9.7 \pm 0.5$ & 1.19 & 8.48 \\
\hline CGPS J001652+642112 & $12.1 \pm 0.5$ & $6.8 \pm 0.5$ & 0.56 & 4.24 \\
\hline CGPS J002359+644044 & $16.0 \pm 0.6$ & $8.7 \pm 0.5$ & 0.59 & 5.27 \\
\hline CGPS J002606+644250 & $9.5 \pm 0.4$ & $2.8 \pm 0.4$ & 1.09 & 5.94 \\
\hline CGPS J002836+631124 & $13.1 \pm 0.5$ & $4.7 \pm 0.5$ & 0.95 & 6.43 \\
\hline CGPS J011455+605142 & $13.6 \pm 0.5$ & $7.4 \pm 0.5$ & 0.59 & 4.56 \\
\hline CGPS J013309+610831 & $9.8 \pm 0.4$ & $17.9 \pm 0.7$ & -0.58 & -4.96 \\
\hline CGPS J020039+650454 & $13.4 \pm 0.5$ & $5.0 \pm 0.5$ & 0.91 & 6.56 \\
\hline CGPS J020222+652747 & $9.9 \pm 0.4$ & $3.3 \pm 0.4$ & 1.00 & 6.07 \\
\hline CGPS J021722+615647 & $34.9 \pm 1.1$ & $17.5 \pm 0.7$ & 0.66 & 7.04 \\
\hline CGPS J022303+612945 & $18.1 \pm 0.8$ & $4.8 \pm 0.5$ & 1.16 & 7.59 \\
\hline CGPS J025804+603843 & $11.2 \pm 0.5$ & $3.9 \pm 0.4$ & 0.96 & 6.14 \\
\hline CGPS J032425+552044 & $14.7 \pm 0.5$ & $7.8 \pm 0.5$ & 0.62 & 5.05 \\
\hline CGPS J032556+554433 & $13.7 \pm 0.5$ & $5.9 \pm 0.5$ & 0.79 & 5.62 \\
\hline CGPS J032641+543419 & $16.8 \pm 0.6$ & $10.8 \pm 0.5$ & 0.43 & 4.23 \\
\hline CGPS J032853+544254 & $4.5 \pm 0.4$ & $10.2 \pm 0.5$ & -0.78 & -4.48 \\
\hline CGPS J032924+531748 & $12.5 \pm 0.5$ & $6.6 \pm 0.5$ & 0.62 & 4.32 \\
\hline CGPS J035853+541313 & $20.4 \pm 1.0$ & $10.0 \pm 0.5$ & 0.68 & 5.09 \\
\hline CGPS J040012+490824 & $4.9 \pm 0.3$ & $9.8 \pm 0.5$ & -0.66 & -4.03 \\
\hline CGPS J040509+510408 & $13.5 \pm 0.5$ & $7.3 \pm 0.5$ & 0.59 & 4.82 \\
\hline CGPS J040916+511511 & $11.6 \pm 0.5$ & $3.2 \pm 0.5$ & 1.13 & 6.39 \\
\hline CGPS J041125+501320 & $6.7 \pm 0.3$ & $2.4 \pm 0.4$ & 0.95 & 4.55 \\
\hline CGPS J041329+514525 & $7.5 \pm 0.3$ & $3.3 \pm 0.4$ & 0.78 & 4.26 \\
\hline
\end{tabular}

Table 3 (continued)

\begin{tabular}{|c|c|c|c|c|}
\hline Name & $\begin{array}{c}\mathrm{S}_{\mathrm{CGPS}} \\
(\mathrm{mJy})\end{array}$ & $\begin{array}{l}\mathrm{S}_{\mathrm{NVSS}} \\
(\mathrm{mJy})\end{array}$ & $\mathrm{F}$ & V \\
\hline CGPS J043122+444202 & $12.8 \pm 0.4$ & $7.3 \pm 0.5$ & 0.54 & 4.45 \\
\hline CGPS J044709+524525 & $7.6 \pm 0.5$ & $2.5 \pm 0.4$ & 1.01 & 4.30 \\
\hline CGPS J045000+524159 & $7.8 \pm 0.5$ & $15.3 \pm 0.6$ & -0.65 & -4.95 \\
\hline CGPS J045000+452234 & $7.7 \pm 0.4$ & $2.2 \pm 0.4$ & 1.11 & 5.41 \\
\hline CGPS J045018+484937 & $10.5 \pm 0.4$ & $5.9 \pm 0.4$ & 0.56 & 4.48 \\
\hline CGPS J045246+430819 & $14.7 \pm 0.5$ & $24.8 \pm 0.8$ & -0.51 & -5.30 \\
\hline CGPS J045808+441958 & $34.6 \pm 1.1$ & $22.0 \pm 0.8$ & 0.45 & 5.20 \\
\hline CGPS J045849+472809 & $7.8 \pm 0.3$ & $13.7 \pm 0.6$ & -0.54 & -4.20 \\
\hline CGPS J050544+360532 & $6.1 \pm 0.4$ & $11.2 \pm 0.5$ & -0.59 & -4.12 \\
\hline CGPS J050749+411450 & $16.3 \pm 0.6$ & $10.0 \pm 0.5$ & 0.48 & 4.47 \\
\hline CGPS J051704+442228 & $16.9 \pm 0.6$ & $25.4 \pm 0.9$ & -0.40 & -3.96 \\
\hline CGPS J052335+405711 & $7.0 \pm 0.3$ & $12.8 \pm 0.6$ & -0.59 & -4.26 \\
\hline CGPS J052902+295502 & $6.4 \pm 0.3$ & $12.9 \pm 0.6$ & -0.67 & -4.83 \\
\hline CGPS J053751+351648 & $7.0 \pm 0.4$ & $13.9 \pm 0.6$ & -0.66 & -4.93 \\
\hline CGPS J054126+240405 & $24.7 \pm 0.8$ & $13.2 \pm 0.6$ & 0.61 & 6.16 \\
\hline CGPS J054519+260810 & $12.3 \pm 0.5$ & $5.0 \pm 0.4$ & 0.84 & 6.34 \\
\hline CGPS J054604+344009 & $25.2 \pm 0.8$ & $38.6 \pm 1.2$ & -0.42 & -4.61 \\
\hline CGPS J054726+225944 & $15.1 \pm 0.5$ & $7.3 \pm 0.5$ & 0.70 & 5.91 \\
\hline CGPS J055034+330022 & $4.4 \pm 0.3$ & $8.9 \pm 0.5$ & -0.68 & -3.89 \\
\hline CGPS J055144+203124 & $12.2 \pm 0.6$ & $21.8 \pm 0.8$ & -0.56 & -4.85 \\
\hline CGPS J055155+235051 & $56.5 \pm 1.7$ & $38.3 \pm 1.2$ & 0.38 & 4.82 \\
\hline CGPS J055206+200719 & $61.4 \pm 1.8$ & $41.3 \pm 1.3$ & 0.39 & 4.89 \\
\hline CGPS J055219+201313 & $9.3 \pm 0.4$ & $16.0 \pm 0.6$ & -0.53 & -4.63 \\
\hline CGPS J055327+223112 & $34.5 \pm 1.1$ & $22.8 \pm 0.8$ & 0.41 & 4.63 \\
\hline CGPS J055532+255852 & $34.8 \pm 1.1$ & $23.3 \pm 0.8$ & 0.40 & 4.64 \\
\hline CGPS J055951+265703 & $10.4 \pm 0.4$ & $16.0 \pm 0.6$ & -0.43 & -3.81 \\
\hline CGPS J060044+254112 & $13.6 \pm 0.5$ & $6.3 \pm 0.5$ & 0.73 & 5.65 \\
\hline CGPS J060046+193941 & $45.9 \pm 1.4$ & $22.8 \pm 0.8$ & 0.67 & 7.61 \\
\hline CGPS J060245+301740 & $6.5 \pm 0.5$ & $14.2 \pm 0.6$ & -0.74 & -5.16 \\
\hline CGPS J060329+194946 & $4.6 \pm 0.3$ & $9.3 \pm 0.5$ & -0.68 & -3.90 \\
\hline CGPS J060542+314556 & $4.5 \pm 0.5$ & $9.9 \pm 0.5$ & -0.75 & -4.04 \\
\hline CGPS J060547+311443 & $5.2 \pm 0.4$ & $11.0 \pm 0.5$ & -0.72 & -4.46 \\
\hline CGPS J060821+282942 & $9.6 \pm 0.4$ & $17.6 \pm 0.7$ & -0.58 & -5.02 \\
\hline CGPS J060838+195646 & $19.2 \pm 0.7$ & $9.7 \pm 0.5$ & 0.66 & 6.09 \\
\hline CGPS J060900+201315 & $35.1 \pm 1.1$ & $22.6 \pm 0.8$ & 0.43 & 4.99 \\
\hline CGPS J061305+200914 & $37.9 \pm 1.2$ & $22.3 \pm 0.8$ & 0.52 & 5.84 \\
\hline CGPS J061420+183700 & $7.7 \pm 0.5$ & $14.0 \pm 0.6$ & -0.58 & -4.10 \\
\hline CGPS J061659+230942 & $25.9 \pm 0.8$ & $6.7 \pm 0.5$ & 1.18 & 10.45 \\
\hline CGPS J061702+233446 & $7.0 \pm 0.3$ & $2.6 \pm 0.4$ & 0.92 & 4.48 \\
\hline CGPS J062652+213422 & $19.9 \pm 0.7$ & $12.8 \pm 0.6$ & 0.43 & 4.21 \\
\hline CGPS J192307+223405 & $5.0 \pm 0.3$ & $9.7 \pm 0.5$ & -0.64 & -4.01 \\
\hline CGPS J192409+252948 & $5.5 \pm 0.3$ & $11.6 \pm 0.5$ & -0.72 & -5.30 \\
\hline CGPS J193124+224331 & $736.6 \pm 21.5$ & $495.0 \pm 14.9$ & 0.39 & 5.04 \\
\hline CGPS J193452+201417 & $4.6 \pm 0.3$ & $9.1 \pm 0.5$ & -0.65 & -3.89 \\
\hline CGPS J193632+220847 & $18.8 \pm 0.7$ & $11.6 \pm 0.5$ & 0.47 & 4.45 \\
\hline CGPS J193633+265436 & $19.2 \pm 0.7$ & $8.5 \pm 0.5$ & 0.77 & 6.77 \\
\hline CGPS J193648+205135 & $58.6 \pm 1.7$ & $30.2 \pm 1.0$ & 0.64 & 7.57 \\
\hline CGPS J193707+311520 & $6.7 \pm 0.4$ & $11.7 \pm 0.5$ & -0.55 & -4.03 \\
\hline CGPS J193939+271240 & $17.5 \pm 0.6$ & $11.4 \pm 0.5$ & 0.42 & 4.39 \\
\hline CGPS J194048+271050 & $8.8 \pm 0.4$ & $3.9 \pm 0.4$ & 0.78 & 4.89 \\
\hline
\end{tabular}


Table 3 (continued)

\begin{tabular}{|c|c|c|c|c|}
\hline Name & $\begin{array}{c}\mathrm{S}_{\mathrm{CGPS}} \\
(\mathrm{mJy})\end{array}$ & $\begin{array}{l}\mathrm{S}_{\mathrm{NVSS}} \\
(\mathrm{mJy})\end{array}$ & $\mathrm{F}$ & $\mathrm{V}$ \\
\hline CGPS J194201+171141 & $24.0 \pm 0.8$ & $40.0 \pm 1.3$ & -0.50 & -5.27 \\
\hline CGPS J194537+325738 & $23.6 \pm 0.7$ & $16.0 \pm 0.6$ & 0.38 & 4.34 \\
\hline CGPS J194728+211157 & $64.2 \pm 1.9$ & $37.4 \pm 1.2$ & 0.53 & 6.46 \\
\hline CGPS J194951+210656 & $207.0 \pm 6.4$ & $138.1 \pm 4.2$ & 0.40 & 4.89 \\
\hline CGPS J195228+372724 & $11.2 \pm 0.5$ & $5.6 \pm 0.5$ & 0.67 & 4.43 \\
\hline CGPS J195737+363830 & $12.2 \pm 0.4$ & $3.9 \pm 0.5$ & 1.03 & 6.70 \\
\hline CGPS J195915+345843 & $17.4 \pm 0.6$ & $7.8 \pm 0.5$ & 0.76 & 6.72 \\
\hline CGPS J200510+364336 & $11.7 \pm 0.4$ & $6.3 \pm 0.5$ & 0.60 & 4.45 \\
\hline CGPS J200702+401729 & $58.6 \pm 2.8$ & $32.9 \pm 1.2$ & 0.56 & 4.61 \\
\hline CGPS J200855+300842 & $7.7 \pm 0.3$ & $13.6 \pm 0.6$ & -0.55 & -4.30 \\
\hline CGPS J200915+403931 & $68.9 \pm 2.8$ & $31.8 \pm 1.1$ & 0.74 & 6.63 \\
\hline CGPS J200921+372739 & $15.8 \pm 0.5$ & $9.1 \pm 0.6$ & 0.54 & 4.54 \\
\hline CGPS J200925+375937 & $29.8 \pm 0.9$ & $16.2 \pm 0.7$ & 0.59 & 6.31 \\
\hline CGPS J200931+372420 & $9.0 \pm 0.4$ & $3.9 \pm 0.5$ & 0.79 & 4.33 \\
\hline CGPS J201008+395741 & $23.5 \pm 1.2$ & $6.6 \pm 0.7$ & 1.12 & 6.49 \\
\hline CGPS J201148+391855 & $21.0 \pm 0.8$ & $9.3 \pm 0.7$ & 0.77 & 5.99 \\
\hline CGPS J201528+412358 & $29.0 \pm 1.0$ & $13.2 \pm 0.7$ & 0.75 & 6.73 \\
\hline CGPS J202036+405756 & $165.9 \pm 5.2$ & $76.8 \pm 2.4$ & 0.73 & 8.29 \\
\hline CGPS J202241+435510 & $29.0 \pm 1.0$ & $13.5 \pm 0.7$ & 0.73 & 6.78 \\
\hline CGPS J202450+370258 & $23.6 \pm 0.8$ & $14.0 \pm 0.6$ & 0.51 & 5.13 \\
\hline CGPS J202516+370920 & $11.6 \pm 0.5$ & $4.3 \pm 0.5$ & 0.92 & 5.74 \\
\hline CGPS J202527+424202 & $14.7 \pm 0.7$ & $3.9 \pm 0.5$ & 1.16 & 6.37 \\
\hline CGPS J202726+380538 & $13.4 \pm 0.5$ & $5.6 \pm 0.6$ & 0.82 & 5.18 \\
\hline CGPS J202751+384325 & $12.7 \pm 0.5$ & $5.9 \pm 0.6$ & 0.73 & 4.55 \\
\hline CGPS J202824+403750 & $39.8 \pm 1.3$ & $12.8 \pm 0.7$ & 1.03 & 9.44 \\
\hline CGPS J202848+324618 & $17.1 \pm 0.8$ & $28.2 \pm 0.9$ & -0.49 & -4.46 \\
\hline CGPS J202924+401114 & $51.6 \pm 1.7$ & $26.1 \pm 1.0$ & 0.66 & 6.89 \\
\hline CGPS J203012+381204 & $11.9 \pm 0.4$ & $3.6 \pm 0.7$ & 1.07 & 5.33 \\
\hline CGPS J203217+372407 & $18.8 \pm 0.6$ & $10.5 \pm 0.6$ & 0.56 & 5.27 \\
\hline CGPS J203227+381010 & $24.6 \pm 0.8$ & $15.2 \pm 0.7$ & 0.47 & 4.80 \\
\hline CGPS J203424+453155 & $14.0 \pm 0.5$ & $2.5 \pm 0.5$ & 1.39 & 8.65 \\
\hline CGPS J203445+363545 & $9.1 \pm 0.3$ & $4.4 \pm 0.5$ & 0.70 & 4.14 \\
\hline CGPS J203827+431329 & $17.6 \pm 0.7$ & $4.4 \pm 0.6$ & 1.20 & 7.89 \\
\hline CGPS J203916+430155 & $26.3 \pm 0.9$ & $17.0 \pm 0.7$ & 0.43 & 4.51 \\
\hline CGPS J204230+404520 & $8.3 \pm 0.4$ & $2.8 \pm 0.6$ & 0.99 & 4.15 \\
\hline CGPS J204258+373537 & $7.0 \pm 0.3$ & $2.7 \pm 0.4$ & 0.88 & 4.25 \\
\hline CGPS J204321+390628 & $21.2 \pm 0.7$ & $12.5 \pm 0.7$ & 0.52 & 4.66 \\
\hline CGPS J204951+424745 & $13.6 \pm 0.6$ & $2.7 \pm 0.5$ & 1.34 & 7.36 \\
\hline CGPS J204955+642258 & $17.2 \pm 0.7$ & $26.0 \pm 0.9$ & -0.41 & -3.83 \\
\hline CGPS J205021+510906 & $15.0 \pm 0.6$ & $9.0 \pm 0.5$ & 0.50 & 4.38 \\
\hline CGPS J205032+390719 & $45.8 \pm 1.4$ & $75.7 \pm 2.3$ & -0.49 & -5.52 \\
\hline CGPS J205205+431013 & $39.2 \pm 1.2$ & $57.4 \pm 1.8$ & -0.38 & -4.10 \\
\hline CGPS J205408+414321 & $25.7 \pm 0.8$ & $37.8 \pm 1.2$ & -0.38 & -4.07 \\
\hline CGPS J205800+421048 & $24.2 \pm 0.8$ & $14.0 \pm 0.6$ & 0.53 & 5.56 \\
\hline CGPS J210511+493405 & $10.2 \pm 0.4$ & $5.2 \pm 0.5$ & 0.65 & 4.25 \\
\hline CGPS J210802+522105 & $8.3 \pm 0.3$ & $3.5 \pm 0.5$ & 0.81 & 4.19 \\
\hline CGPS J210821+502815 & $21.2 \pm 0.8$ & $13.1 \pm 0.6$ & 0.47 & 4.58 \\
\hline CGPS J210905+522627 & $14.7 \pm 0.6$ & $8.4 \pm 0.5$ & 0.55 & 4.45 \\
\hline CGPS J211955+492245 & $11.7 \pm 0.4$ & $6.3 \pm 0.5$ & 0.60 & 4.36 \\
\hline CGPS J212331+522844 & $14.1 \pm 0.6$ & $7.6 \pm 0.5$ & 0.60 & 4.54 \\
\hline
\end{tabular}

Table 3 continued
Table 3 (continued)

\begin{tabular}{|c|c|c|c|c|}
\hline Name & $\begin{array}{c}\mathrm{S}_{\mathrm{CGPS}} \\
(\mathrm{mJy})\end{array}$ & $\begin{array}{r}\mathrm{S}_{\mathrm{NVSS}} \\
(\mathrm{mJy})\end{array}$ & $\mathrm{F}$ & $\mathrm{V}$ \\
\hline CGPS J212511+574220 & $23.5 \pm 0.9$ & $36.4 \pm 1.2$ & -0.43 & -4.23 \\
\hline CGPS J213835+500901 & $18.9 \pm 0.6$ & $10.0 \pm 0.6$ & 0.62 & 5.60 \\
\hline CGPS J214148+545858 & $12.8 \pm 0.5$ & $6.8 \pm 0.5$ & 0.61 & 4.68 \\
\hline CGPS J215403+531840 & $4.3 \pm 0.3$ & $9.1 \pm 0.5$ & -0.71 & -4.03 \\
\hline CGPS J221021+525433 & $8.4 \pm 0.4$ & $3.8 \pm 0.4$ & 0.76 & 4.34 \\
\hline CGPS J224530+701557 & $10.9 \pm 0.5$ & $6.0 \pm 0.4$ & 0.58 & 4.20 \\
\hline CGPS J224645+580306 & $32.7 \pm 1.1$ & $21.2 \pm 0.8$ & 0.43 & 4.64 \\
\hline CGPS J225359+644422 & $11.9 \pm 0.5$ & $6.3 \pm 0.5$ & 0.62 & 4.47 \\
\hline CGPS J225522+581311 & $8.8 \pm 0.4$ & $2.4 \pm 0.5$ & 1.14 & 5.18 \\
\hline CGPS J230114+572047 & $6.6 \pm 0.3$ & $2.3 \pm 0.4$ & 0.96 & 4.41 \\
\hline CGPS J230948+622935 & $13.0 \pm 0.5$ & $7.4 \pm 0.5$ & 0.55 & 4.42 \\
\hline CGPS J233748+655723 & $10.4 \pm 0.4$ & $5.0 \pm 0.5$ & 0.70 & 4.36 \\
\hline CGPS J234042+614508 & $9.4 \pm 0.4$ & $3.2 \pm 0.6$ & 0.99 & 4.65 \\
\hline CGPS J234205+613806 & $8.9 \pm 0.4$ & $3.3 \pm 0.5$ & 0.92 & 4.79 \\
\hline CGPS J234210+613508 & $14.8 \pm 0.5$ & $5.8 \pm 0.6$ & 0.87 & 6.13 \\
\hline CGPS J234612+610229 & $15.4 \pm 0.5$ & $7.3 \pm 0.5$ & 0.72 & 6.08 \\
\hline CGPS J235007+601852 & $9.2 \pm 0.4$ & $2.6 \pm 0.4$ & 1.11 & 5.96 \\
\hline CGPS J235032+643035 & $12.5 \pm 0.5$ & $6.4 \pm 0.5$ & 0.64 & 4.70 \\
\hline CGPS J235221+590043 & $7.7 \pm 0.4$ & $2.8 \pm 0.4$ & 0.94 & 4.86 \\
\hline CGPS J235312+644017 & $9.2 \pm 0.4$ & $3.3 \pm 0.4$ & 0.95 & 5.69 \\
\hline CGPS J235400+594207 & $7.3 \pm 0.3$ & $3.2 \pm 0.4$ & 0.78 & 4.19 \\
\hline CGPS J235612+625132 & $7.4 \pm 0.4$ & $3.1 \pm 0.4$ & 0.82 & 4.26 \\
\hline
\end{tabular}

In addition to searching for variable sources that appear in both the CGPS and NVSS catalogues, we also looked for candidate transient objects that appear in the CGPS but have no counterpart in the NVSS catalog. There are 19,911 sources in the CGPS without NVSS counterparts. The vast majority of these are faint objects below $\sim 3 \mathrm{mJy}$ that are missed by the NVSS due to the high level of NVSS imaging artefacts arising from the lack of low spatial frequency data in the presence of the strong extended emission at low Galactic latitudes. This effect is illustrated in Figure 7, where we compare CGPS and NVSS images for one of the CGPS $5.12^{\circ} \times 5.12^{\circ}$ mosaics. These images include Cas A, and artefacts from this strong source are visible in both. The diffuse structure away from Cas A visible in the CGPS is not well represented in the NVSS image and results in lower level artefacts in the NVSS that extend throughout the image. The CGPS fully samples the structure of the diffuse emission resulting in much greater intensity dynamic range. The CGPS fully samples the structure of the diffuse emission resulting in much greater intensity dynamic range.

To avoid missing sources due to the effect described above, we limited the analysis for candidate transients to unresolved sources stronger than $10 \mathrm{mJy}$ in the CGPS. The resulting 71 objects were each examined in the 

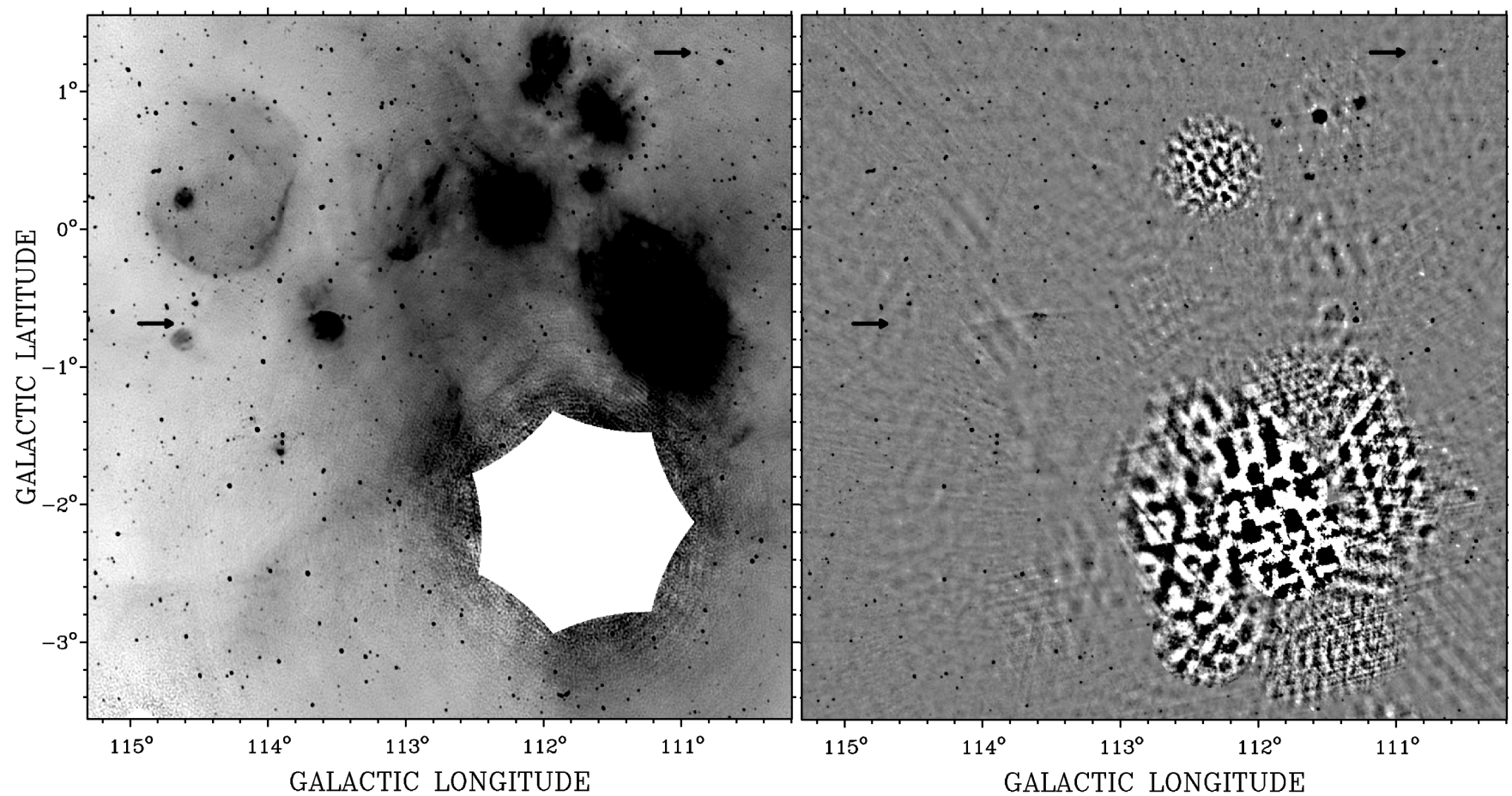

Figure 7. Comparison of CGPS (left) and NVSS (right) images of a field near Cas A. The area around Cas A is blanked in the CGPS image. The grayscale in the CGPS images extends from $5 \mathrm{~K}$ (white) to $8 \mathrm{~K}$ (black), and in the NVSS image from -0.015 (white) to +0.015 (black) mJy/beam. Two sources that are possibly transients are marked by arrows in the CGPS image; they cannot be detected in the NVSS image (see Figure 8).

NVSS images. In most cases, the missing detections in the NVSS could be attributed to strong image artefacts obscuring the source. The final list of 13 transient candidates includes sources that should have been visible in the NVSS if present at the CGPS flux levels. These sources are listed in Table 4. Figure 8 shows an example of one transient candidate, CGPS J234153+610329, that is detected at $12.6 \mathrm{mJy}$ in the CGPS but is not visible in the NVSS down to the NVSS image RMS at the source location of $0.58 \mathrm{mJy}$.
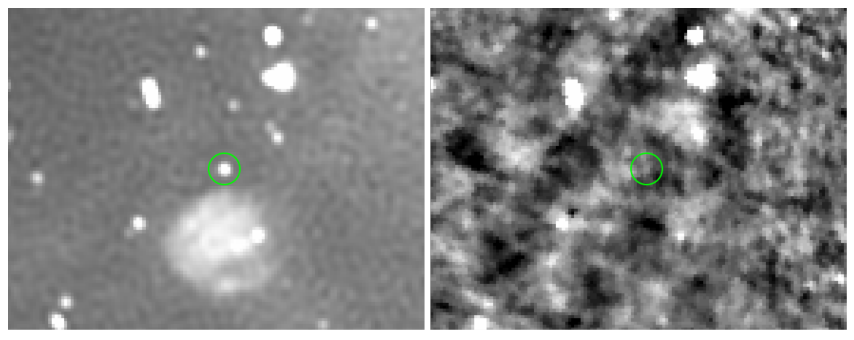

Figure 8. A close up of CGPS (left) and NVSS (right) of the area of the transient candidate CGPS J234153+610329. The object indicated by the circle has flux density of $12.6 \mathrm{mJy}$ in the CGPS catalogue. There is no evidence of the source in the NVSS image, which has an RMS in the location of the source of $0.58 \mathrm{mJy}^{\text {beam }}{ }^{-1}$.
Table 4. Strong CGPS sources not detected in the NVSS

\begin{tabular}{ccc}
\hline \hline Name & $\begin{array}{c}\mathrm{S}_{\text {CGPS }} \\
(\mathrm{mJy})\end{array}$ & Comment \\
\hline CGPS J200258+333748 & $18.5 \pm 0.6$ & weak NVSS source visible \\
CGPS J201912+420531 & $24.8 \pm 0.9$ & \\
CGPS J201936+405852 & $20.2 \pm 1.5$ & \\
CGPS J202626+394015 & $17.8 \pm 0.8$ & \\
CGPS J202635+421335 & $18.7 \pm 0.7$ & weak NVSS source visible \\
CGPS J202701+354228 & $10.0 \pm 0.5$ & \\
CGPS J203245+384529 & $16.2 \pm 0.6$ & weak NVSS source visible \\
CGPS J203315+463424 & $12.5 \pm 0.6$ & weak NVSS source visible \\
CGPS J221640+561817 & $10.4 \pm 0.4$ & weak NVSS source visible \\
CGPS J224712+580953 & $22.7 \pm 0.9$ & weak NVSS source visible \\
CGPS J225759+623319 & $24.6 \pm 1.4$ & weak NVSS source visible \\
CGPS J230704+614118 & $13.0 \pm 0.5$ & \\
CGPS J234153+610329 & $12.6 \pm 0.4$ & \\
\hline
\end{tabular}

\section{CONCLUSIONS}

We have described a catalog of 72,787 compact sources down to a minimum peak total intensity of $\sim 1 \mathrm{mJy}$ from the Canadian Galactic Plane Survey, and have given details of electronic access to it. The catalog presents po- 
sition, total intensity and linearly polarized intensity, polarization position angle, and source size. The flux density scale and the positions of the CGPS were tied to the VLA Northern Sky Survey catalog using strong, isolated CGPS sources that have counterparts in the NVSS. The positions in the two catalogs are aligned to systematic error of less than $\sim 0.01^{\prime \prime}$, and flux densities to within $0.5 \%$. We have detected polarization from 12,368 sources.

The effects of bandwidth depolarization are substantially smaller in the CGPS than in the NVSS, and the CGPS catalog provides a superior list of polarized intensities at low Galactic latitudes, where the NVSS polarized intensities are significantly affected by depolarization. Since the CGPS and the NVSS were observed at different times, we have used the comparison to identify variable and possibly transient sources, and list 149 variables that are detected in both surveys, and 13 candidate transient sources detected in the CGPS but missing in the NVSS.

The Dominion Radio Astrophysical Observatory is operated as a national facility by the National Research Council Canada. The Canadian Galactic Plane Survey was supported by the Natural Sciences and Engineering Research Council. The high quality of the CGPS data owes much to the excellent work of the DRAO staff who made the observations and maintained the telescope, and that of the CGPS team who reduced the data.

Facility: DRAO (synthesis telescope)

\section{REFERENCES}

Brown, J. C., Taylor, A. R., \& Jackel, B. J. 2003, ApJS, 145, 213

Condon, J. J., Cotton, W. D., Greisen, E. W., et al. 1998, AJ, 115,1693

Higgs, L. A., Hoffmann, A. P., \& Willis, A. G. 1997, in Astronomical Society of the Pacific Conference Series, Vol. 125, Astronomical Data Analysis Software and Systems VI, ed. G. Hunt \& H. Payne, 58
Landecker, T. L., Dewdney, P. E., Burgess, T. A., et al. 2000, A\&AS, 145,509

Landecker, T. L., Reich, W., Reid, R. I., et al. 2010, A\&A, 520, A80

Simmons, J. F. L., \& Stewart, B. G. 1985, A\&A, 142, 100

Taylor, A. R., Stil, J. M., \& Sunstrum, C. 2009, ApJ, 702, 1230

Taylor, A. R., Gibson, S. J., Peracaula, M., et al. 2003, AJ, 125, 3145 